\title{
Publicações científicas e acesso a mestrados de alunos de Iniciação Científica: um estudo nos cursos do Setor de Saúde da Universidade Federal do Paraná
}

\author{
Felipe Correa Fiori \\ Mestre; Universidade Federal do Paraná, Curitiba, PR, Brasil; \\ felipefiori08@gmail.com \\ Márcio Rogério de Souza \\ Mestre; Universidade Federal do Paraná, Curitiba, PR, Brasil; \\ mrsouza.cwb@gmail.com

\section{Cicero Aparecido Bezerra} \\ Doutor; Universidade Federal do Paraná, Curitiba, PR, Brasil; \\ cicero.bezerra@ufpr.br
}

\begin{abstract}
Resumo: O estudo busca relações entre publicações científicas e ingresso em programas de mestrado de 809 egressos de Iniciação Científica dos cursos do Setor de Saúde da Universidade Federal do Paraná (Enfermagem, Farmácia, Medicina, Nutrição, Odontologia e Terapia Ocupacional), entre 2002 e 2017. Trata-se de um estudo que emprega análises estatísticas bivariadas não paramétricas em dados coletados do Sistema de Iniciação Científica da Universidade Federal do Paraná e da plataforma Lattes. Os resultados mostram que egressos que permaneceram por mais tempo em IC se tornaram mestrandos mais rapidamente, ao mesmo tempo em que apresentaram maior número de publicações; além disso, ex-alunos dos cursos de Odontologia e Enfermagem obtiveram um número médio de publicações acima do dobro dos demais cursos, bem como os egressos de Farmácia e Enfermagem seguiram mais rapidamente a carreira acadêmica.
\end{abstract}

Palavras-chave: Iniciação Científica. Ciências da Saúde. Publicações científicas. Mestrados.

\section{Introdução}

A importância da pesquisa em Saúde, em um contexto nacional, é indiscutível. Em 2015, o Ministério da Saúde do Brasil já visualizava a pesquisa em Saúde como um "[...] processo político que busca, em todas as suas etapas, a ampla 
participação de atores com experiências e linguagens distintas tanto da pesquisa como da saúde" (BRASIL. MINISTÉRIO DA SAÚDE, 2015, p. 5).

Assim sendo, é preciso apoiar os agentes inseridos no contexto econômico, político, social e cultural voltados à pesquisa científica e tecnológica (SANTOS et al., 2017). Entre esses agentes, as universidades possuem especial destaque, haja vista sua capacidade de valorização e incentivo aos atores envolvidos em atividades de pesquisa (SILVA JUNIOR et al., 2014).

Nas universidades, o primeiro contato com a pesquisa ocorre em programas de Iniciação Científica (IC). Para Santos e Leal (2014, p. 31)

a participação em projetos de IC proporciona aos estudantes o contato com a produção científica e possibilita o desenvolvimento de competências, habilidades e conhecimentos científicos, engajando-os em atividades de investigação.

A observação de Santos e Leal (2014) ganha especial destaque nas Ciências da Saúde, uma vez que os avanços científicos nesta área provocam impactos não somente no processo pedagógico dos estudantes, como também na própria abordagem clínica (SOARES et al., 2017; FIGUEIREDO et al., 2018). Nesse contexto, autores como Erdmann et al. (2011), Santos, Anjos e Almeida (2015), Silva et al. (2017), Rocha et al. (2019) e Pirola et al. (2020) pontuam a relevância do desenvolvimento de estudos que abordem o incentivo da pesquisa científica no âmbito da graduação, não somente pelo impacto que esse tipo de atividade exerce na construção da vida acadêmica, mas na própria formação profissional.

Porém, em que pese a importância da relação da IC na área da Saúde, é premente a necessidade de se desenvolverem mais estudos: segundo Massi e Queiroz (2015), apesar da abrangência e do crescimento apresentado pela IC, poucas pesquisas foram desenvolvidas no sentido de se perceber os efeitos para a formação do graduando; Pinto, Fernandes e Silva (2016) entendem que, na literatura especializada, ainda são poucos os estudos sobre o impacto gerado pela IC na produção acadêmica de instituições; para Pinho (2017), é necessário estudar caminhos que apontem e ampliem o entendimento sobre as contribuições advindas da prática de IC dentro das instituições que a executam; Erdmann et al. (2011) vão além ao categorizar como necessários estudos 
envolvendo a IC e seus atores, visando a otimizar os investimentos e recursos públicos destinados à atividade.

Esse é o contexto no qual o presente estudo se enquadra, ao responder o seguinte problema de pesquisa: existem relações entre as características de egressos de IC de cursos da área da Saúde da Universidade Federal do Paraná (UFPR) quanto à produção científica e sua inserção em programas de mestrados?

Para responder à questão norteadora, o estudo teve como objetivo principal identificar padrões entre a atividade de IC associadas às publicações científicas desenvolvidas pelos estudantes e o ingresso destes em cursos de mestrado. Para atingir o objetivo principal, foram definidos como objetivos específicos: a caraterização da atividade de IC em âmbito nacional e relatos da atividade na área da saúde contidos na literatura, a coleta das informações nas fontes de dados definidas e a aplicação de métodos estatísticos que permitam responder ao problema de pesquisa proposto e às relações subliminares evidenciadas apenas no momento das análises.

Trata-se de uma pesquisa quantitativa, com técnicas estatísticas bivariadas não paramétricas empregadas à população de egressos do Programa Institucional de Bolsas de Iniciação Científica (PIBIC) da IC entre 2002 e 2017, para os cursos de Enfermagem, Farmácia, Medicina, Nutrição, Odontologia e Terapia Ocupacional, do Setor de Ciências da Saúde da UFPR, com dados coletados no Sistema de Iniciação Científica e Tecnológica da UFPR e na Plataforma Currículo Lattes do Conselho Nacional de Pesquisa (CNPq).

\section{Iniciação Científica}

A IC pode ser definida como a "vivência prática no processo da pesquisa", representando o caminho inicial do estudante de graduação na ciência, tecnologia e pesquisa por meio da orientação de um professor de sua instituição (ROCHA et al., 2019, p. 5447). Segundo Massi e Queiroz (2015, p. 7), "[...] é um processo que abarca todas as experiências vivenciadas pelo aluno durante a graduação, com o objetivo de promover o seu envolvimento com a pesquisa". 
No Brasil, a IC teve sua origem em experiências institucionalizadas nos Estados Unidos e na França, tendo sido consolidada com a fundação do Conselho Nacional de Desenvolvimento Científico e Tecnológico (CNPq), em 1951 (BAZIN, 1983). Em 1988, o governo brasileiro optou por fomentar a IC em Instituições de Ensino Superior (IESs) e em outras organizações onde se realiza pesquisa por meio do PIBIC, que é considerado por Santos et al. (2017), o maior programa de IC em valores brutos. Por sua vez, a UFPR data relatos de pesquisa antes mesmo da institucionalização da IC pelo país, com destaque para a área de Ciências da Saúde (UNIVERSIDADE FEDERAL DO PARANÁ, 1998). Com o maior alcance do PIBIC, a UFPR também se beneficiou deste programa, sendo considerado um dos fatores essenciais para a ampliação e consolidação da IC na instituição (UNIVERSIDADE FEDERAL DO PARANÁ, 1998).

Com base na resolução normativa 017/2006 do CNPq, os objetivos do PIBIC podem ser classificados de acordo com os atores envolvidos no processo (CONSELHO NACIONAL DE DESENVOLVIMENTO CIENTÍFICO E TECNOLÓGICO, 2006):

- Bolsistas: formação de recursos humanos para a pesquisa, contribuindo para reduzir o tempo médio de titulação dos estudantes na graduação;

- Orientadores: envolvimento de estudantes de graduação nas atividades científica, tecnológica, profissional e artístico-cultural;

- Instituições: formulação de uma política de IC, possibilitando maior interação entre a graduação e a pós-graduação.

Pode-se afirmar que há consenso de que, na graduação, a IC é considerada a ferramenta que propicia habilidades ao estudante de maneira mais eficaz, desenvolvendo competências diferenciadas, criando atitudes de independência e autonomia ante o conhecimento e as questões do cotidiano, colaborando para formar um profissional mais autônomo, independente e de destaque em sua área de atuação, principalmente quando comparado àqueles alunos que não participaram desta experiência (LOPES e SOUSA JÚNIOR, 
2018; ZAMPIERI et al., 2018; NARDINI et al., 2019). A atividade passa a ser também uma grande oportunidade aos seus participantes que pretendem seguir a carreira acadêmica, possibilitando aos mesmos fazer parte do processo de produção de conhecimento em sua área de formação ou de interesse, desenvolvendo publicações científicas ainda na graduação (SOARES, et al., 2017; ROCHA, et al., 2019; NARDINI, et al., 2019). Ainda que diversos benefícios sejam associados à prática da IC, a atividade apresenta dificuldades. Autores como Silva Junior et al. (2014) e Pinto, Fernandes e Silva (2016) evidenciam as principais dificuldades apontadas por participantes de IC: tempo escasso para desenvolver a pesquisa; falta de aporte financeiro e pouca infraestrutura disponível para alguns tipos de estudos. A incapacidade de condução do projeto por parte dos orientadores, o corte de bolsas e a falta de divulgação e esclarecimento por parte das IES também são citados por Rocha e Rodrigues (2017). Nesse sentido, Pirola et al. (2020) apontam que a fraca divulgação da IC promove o desconhecimento para os jovens discentes dos benefícios que a atividade promove e também gera insegurança face aos conhecimentos prévios necessários.

A IC é caracterizada também por oferecer auxílio financeiro ao participante por meio de bolsas (PINHO, 2017). Apesar de este conceito estar intimamente ligado ao fornecimento de recursos financeiros, são comuns estudantes trabalhando de forma voluntária e realizando, em geral, as mesmas tarefas designadas a um bolsista, sendo notável o empenho demonstrado por esses indivíduos para com a pesquisa à qual estão vinculados (CABRERO; COSTA, 2015). No Brasil, apesar de o número de bolsas vir aumentando ao longo dos anos, os programas de IC alcançam apenas 0,4\% dos ingressantes na graduação, sendo constante o debate a respeito da sua abrangência, principalmente para as regiões Norte e Centro-Oeste do país (MASSI; QUEIROZ, 2015). 


\section{Material e métodos}

O estudo foi efetuado sobre os dados secundários de todos os participantes na IC dos cursos do Setor de Ciências da Saúde (SCS) da UFPR (Enfermagem, Farmácia, Medicina, Nutrição, Odontologia e Terapia Ocupacional), egressos entre os anos de 2002 e 2017, coletados junto ao Sistema de Iniciação Científica e Tecnológica (SICT) da UFPR e à plataforma Lattes. O recorte temporal devese à disponibilidade das informações do SICT, que iniciou os registros dos dados de maneira sistematizada a partir de 2002. As variáveis coletadas são apresentadas no Quadro 1:

Quadro 1 - Variáveis de análise

\begin{tabular}{|c|c|c|}
\hline Tipo & Variável & Natureza \\
\hline \multirow{9}{*}{  } & Idade & Numérica intervalar, representando a idade do bolsista em anos. \\
\hline & Sexo & Categórica, com as opções "Masculino" e "Feminino". \\
\hline & Curso & $\begin{array}{l}\text { Categórica, com as opções "Enfermagem", "Farmácia”, "Odontologia”, } \\
\text { "Nutrição", "Medicina" e "Terapia Ocupacional". }\end{array}$ \\
\hline & $\begin{array}{l}\text { Tipo de } \\
\text { participação }\end{array}$ & $\begin{array}{l}\text { Categórica, com as opções "CNPq", "UFPR/TN", "Fundação Araucária", "Mais } \\
\text { de um tipo de participação" (participações remuneradas). Além disso, } \\
\text { registraram-se as participações "Voluntária" e "Mais de um tipo de participação e } \\
\text { voluntária". }\end{array}$ \\
\hline & $\begin{array}{l}\text { Tempo } \\
\text { permanecido }\end{array}$ & $\begin{array}{l}\text { Numérica intervalar, representando o número total de meses que o participante } \\
\text { permaneceu na IC. }\end{array}$ \\
\hline & Carga horária total & $\begin{array}{l}\text { Numérica intervalar, representando o número total de horas estipuladas para o } \\
\text { desenvolvimento do projeto. }\end{array}$ \\
\hline & Total recebido & $\begin{array}{l}\text { Numérica intervalar, representando o montante financeiro recebido pelo } \\
\text { participante enquanto se manteve em IC. }\end{array}$ \\
\hline & $\begin{array}{l}\text { Número } \\
\text { participações }\end{array}$ & $\begin{array}{l}\text { Numérica intervalar, representando o número de participações distintas em } \\
\text { atividades de IC. }\end{array}$ \\
\hline & $\begin{array}{l}\text { Número } \\
\text { orientadores }\end{array}$ & $\begin{array}{l}\text { Numérica intervalar, representando o número de orientadores distintos nas } \\
\text { atividades de IC. }\end{array}$ \\
\hline \multirow{5}{*}{ 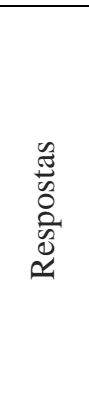 } & $\begin{array}{l}\text { Ingresso em } \\
\text { mestrado }\end{array}$ & Categórica, com as opções "Sim" e "Não". \\
\hline & Tipo IES mestrado & $\begin{array}{l}\text { Categórica, com as opções "UFPR", "Federal", "Estadual", "Privada" e } \\
\text { "Internacional". }\end{array}$ \\
\hline & $\begin{array}{l}\text { Tempo para } \\
\text { ingresso }\end{array}$ & $\begin{array}{l}\text { Numérica intervalar, representando o tempo em anos para o ingresso no mestrado } \\
\text { após o fim da graduação. }\end{array}$ \\
\hline & $\begin{array}{l}\text { Publicações } \\
\text { durante IC }\end{array}$ & $\begin{array}{l}\text { Numérica intervalar, representando o somatório de publicações do aluno durante } \\
\text { o período vivenciado na IC, independente da ordem de autoria ou estrato. }\end{array}$ \\
\hline & $\begin{array}{l}\text { Publicações após } \\
\text { IC }\end{array}$ & $\begin{array}{l}\text { Numérica intervalar, representando a quantidade total de publicações do aluno no } \\
\text { período após sua saída da IC, independente da ordem de autoria ou estrato. }\end{array}$ \\
\hline
\end{tabular}
Fonte: elaborado pelos autores (2020).

O objetivo da presença das variáveis explicativas é verificar se estas se encontram associadas às variáveis respostas (ambas mostradas no Quadro 1), de tal forma a responder o problema de pesquisa inicialmente proposto. 
Os dados associados a cada variável foram obtidos a partir do protocolo mostrado no Quadro 2:

Quadro 2 - Protocolo de coleta de dados

\begin{tabular}{|c|c|c|c|}
\hline Etapa & Fonte de dados & Dados coletados/gerados & Método para coleta/geração \\
\hline 1 & $\begin{array}{l}\text { Banco de dados } \\
\text { relacional MySQL do } \\
\text { Sistema de Iniciação } \\
\text { Científica e Tecnológica } \\
\text { (SICT) UFPR } \\
\text { (https://www.prppg.ufpr. } \\
\text { br/sict/login.xhtml), } \\
\text { responsável por gerenciar } \\
\text { processos e informações } \\
\text { relacionados aos editais } \\
\text { de IC. }\end{array}$ & $\begin{array}{l}\text { Ano nascimento (empregado } \\
\text { para gerar a variável "Idade”), } \\
\text { Sexo, Curso, Tipo de } \\
\text { participação, Tempo } \\
\text { permanecido, Carga horária } \\
\text { total e Total recebido. }\end{array}$ & $\begin{array}{l}\text { Coleta realizada por meio de queries } \\
\text { de consultas MySQL, em dados } \\
\text { armazenados pelo SICT que, } \\
\text { posteriormente são inseridos em uma } \\
\text { nova tabela, a partir de query de } \\
\text { criação de tabelas. }\end{array}$ \\
\hline 2 & $\begin{array}{l}\text { Currículo Lattes } \\
\text { (http://buscatextual.cnpq. } \\
\text { br/buscatextual/busca.do? } \\
\text { metodo=apresentar). }\end{array}$ & $\begin{array}{l}\text { Ano de término da graduação, } \\
\text { Ano de ingresso em mestrado } \\
\text { (empregadas para gerar as } \\
\text { variáveis "Ingresso em } \\
\text { mestrado" e "Tempo para } \\
\text { ingresso"), Publicações } \\
\text { (empregada para gerar as } \\
\text { variáveis "Publicações durante } \\
\text { IC" e "Publicações após IC") }\end{array}$ & $\begin{array}{l}\text { O servidor que mantém o Sistema de } \\
\text { Gestão Acadêmica da UFPR (SIGA- } \\
\text { UFPR) possui acesso ao webservice } \\
\text { que o CNPq dispõe para instituições } \\
\text { parceiras consultarem a Plataforma } \\
\text { Lattes. Esse serviço, por sua vez, tem } \\
\text { como base o procedimento remoto } \\
\text { XML-RPC, que utiliza o protocolo } \\
\text { HTTP como transporte e o XML } \\
\text { como codificação dos dados (XML- } \\
\text { RPC, 2020). Foram empregadas } \\
\text { queries de consulta MySQL para } \\
\text { seleção dos dados e de criação, } \\
\text { inserção e atualização de dados na } \\
\text { tabela criada na Etapa } 1 .\end{array}$ \\
\hline 3 & $\begin{array}{l}\text { Tabela de dados criada e } \\
\text { atualizada nas Etapas } 1 \text { e } \\
2 .\end{array}$ & $\begin{array}{l}\text { Número de participações, } \\
\text { Número de orientadores. }\end{array}$ & $\begin{array}{l}\text { Empregaram-se scripts PHP e } \\
\text { queries SQL de seleção e atualização } \\
\text { para totalizar e consolidar as } \\
\text { informações obtidas ao longo do } \\
\text { tempo em torno de um registro único } \\
\text { por participante de IC. }\end{array}$ \\
\hline
\end{tabular}

Fonte: elaborado pelos autores (2020).

Os dados coletados foram submetidos ao protocolo de análise mostrado no Quadro 3:

\begin{tabular}{|c|l|l|}
\hline \multicolumn{4}{|c|}{ Quadro 3 - Protocolo de análise } \\
\hline Etapa & Procedimentos & Objetivos \\
\hline 1 & $\begin{array}{l}\text { Estatísticas descritivas: médias, } \\
\text { desvios padrão, mínimos, } \\
\text { máximos, frequências. }\end{array}$ & $\begin{array}{l}\text { Descrever o conjunto de dados a partir das variáveis coletadas } \\
\text { (MATLOFF, 2019; OGLE, WHEELER e DINNO, 2019). }\end{array}$ \\
\hline 2 & $\begin{array}{l}\text { Tabelas de contingência: qui- } \\
\text { quadrado }\left(X^{2}\right) \text { de Pearson. }\end{array}$ & $\begin{array}{l}\text { Avaliar a independência entre pares de variáveis categóricas } \\
\text { (PEARSON 1900; FIELD, MILES e FIELD, 2012). }\end{array}$ \\
\hline 3 & Teste W de Shapiro-Francia. & $\begin{array}{l}\text { Avaliar a existência do pressuposto paramétrico de normalidade } \\
\text { SHAPIRO E FRANCIA 1972; ANDERSON, et al., 2017; } \\
\text { GROSS E LIGGES, 2020). }\end{array}$ \\
\hline 4 & $\begin{array}{l}\text { Teste W de Wilcoxon-Mann- } \\
\text { Whitney. }\end{array}$ & $\begin{array}{l}\text { Verificar a igualdade de medianas de variável numérica entre dois } \\
\text { grupos de variável categórica (WILCOXON, 1945; MANN e }\end{array}$ \\
\hline
\end{tabular}




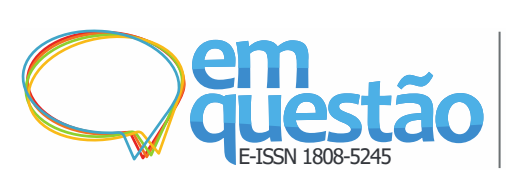

Publicações científicas e acesso a mestrados de alunos de Iniciação Científica: um estudo nos cursos do Setor de

Saúde da Universidade Federal do Paraná

Felipe Correa Fiori, Márcio Rogério de Souza, Cicero Aparecido Bezerra

\begin{tabular}{|c|l|l|}
\hline & & WHITNEY, 1947; KLOKE e MCKEAN, 2014). \\
\hline 5 & Teste $X^{2}$ de Kruskal-Wallis. & $\begin{array}{l}\text { Verificar a igualdade de medianas de variável numérica entre } \\
\text { mais de dois grupos de variável categórica (KRUSKAL e } \\
\text { WALLIS, 1952; KRASKA-MULLER, 2019). }\end{array}$ \\
\hline 6 & Teste rho de Spearman. & $\begin{array}{l}\text { Medir o grau de associação entre duas variáveis numéricas } \\
\text { (SPEARMAN, 1904; KING e ECKERSLEY, 2019). }\end{array}$ \\
\hline
\end{tabular}

Fonte: elaborado pelos autores (2020).

A organização e a tabulação dos dados foram efetuadas no software Microsoft Excel 2013, enquanto que as análises foram efetuadas no software R em sua versão 3.6.1, sendo que, nos casos onde foi necessário, empregou-se um intervalo de confiança de 95\% e um nível esperado de significância de 0,05.

\section{Apresentação, análise e discussão de resultados}

Registraram-se no SCS 809 alunos que participaram de atividades de IC durante o período compreendido entre 2002 e 2017. Os percentuais de alunos, nessas condições, nos cursos ficaram assim distribuídos: Medicina (34,73\%), Farmácia (23,24\%), Enfermagem (16,81\%), Odontologia (11,99\%), Nutrição $(8,53 \%)$ e Terapia Ocupacional (4,70\%). Em relação ao sexo, a população se dividiu em $77,99 \%$ de mulheres e $22,01 \%$ de homens.

A Tabela 1 mostra, a seguir, as características gerais da população estudada, apresentadas a partir de estatísticas descritivas:

\begin{tabular}{|c|c|c|c|c|}
\hline Variável & média & desvio & mínimo & máximo \\
\hline Idade (anos) & 23,78 & 2,91 & 19 & 50 \\
\hline Tempo permanecido (meses) & 14,54 & 8,06 & 1 & 60 \\
\hline Total recebido $(\mathrm{R} \$)$ & $3.622,69$ & 3219,00 & 0 & $20.520,00$ \\
\hline Carga horária total (horas) & 949,87 & 635,49 & 48 & 4352 \\
\hline Número participações & 1,60 & 0,88 & 1 & 6 \\
\hline Número orientadores & 1,13 & 0,34 & 1 & 4 \\
\hline
\end{tabular}

Fonte: dados da pesquisa (2020).

Quanto ao encaminhamento para a pós-graduação, observou-se que 29,91\% dos acadêmicos posteriormente à sua participação ingressaram em um curso de mestrado, levando em média 2,1 anos para o ingresso, com desvio padrão de 2,15 anos, após a conclusão da graduação - houve casos de alunos que foram para o mestrado no mesmo ano de conclusão do curso e o caso de um egresso que levou 12 anos para ingressar em mestrado. Dos que seguiram a carreira acadêmica, 79,34\% o fizeram na própria instituição; 11,69\% em outras 
instituições federais; $6,61 \%$ em universidades estaduais; $1,65 \%$ em universidades privadas; e 1,24\% em instituições internacionais.

Para os números de publicações científicas realizadas por esses alunos, a Tabela 2 apresenta as estatísticas descritivas para as publicações totais do aluno, durante e após a IC:

Tabela 2 - Publicações científicas

\begin{tabular}{lrrrrr} 
Variável & média & desvio & mínimo & máximo & Total \\
\hline Durante IC & 4,98 & 8,44 & 0 & 110 & 4.033 \\
Pós-IC & 9,67 & 19,31 & 0 & 242 & 7.825 \\
\hline \multicolumn{5}{c}{ Fonte: dados da pesquisa (2020). }
\end{tabular}

É importante ressaltar que $24,22 \%$ dos discentes participantes não realizaram nenhuma publicação de única autoria e 35,97\% não publicaram nada após terem concluído as atividades de IC.

Seguindo o protocolo de análise, ao se efetuar o cruzamento das variáveis "Sexo" e "Ingresso em mestrado", o teste do $\mathrm{X}^{2}$ de Pearson não evidenciou diferenças estatisticamente significativas $\left(\mathrm{X}^{2}(1)=0,773\right.$, p-valor < 0,379), conforme visualizado na Figura 1:

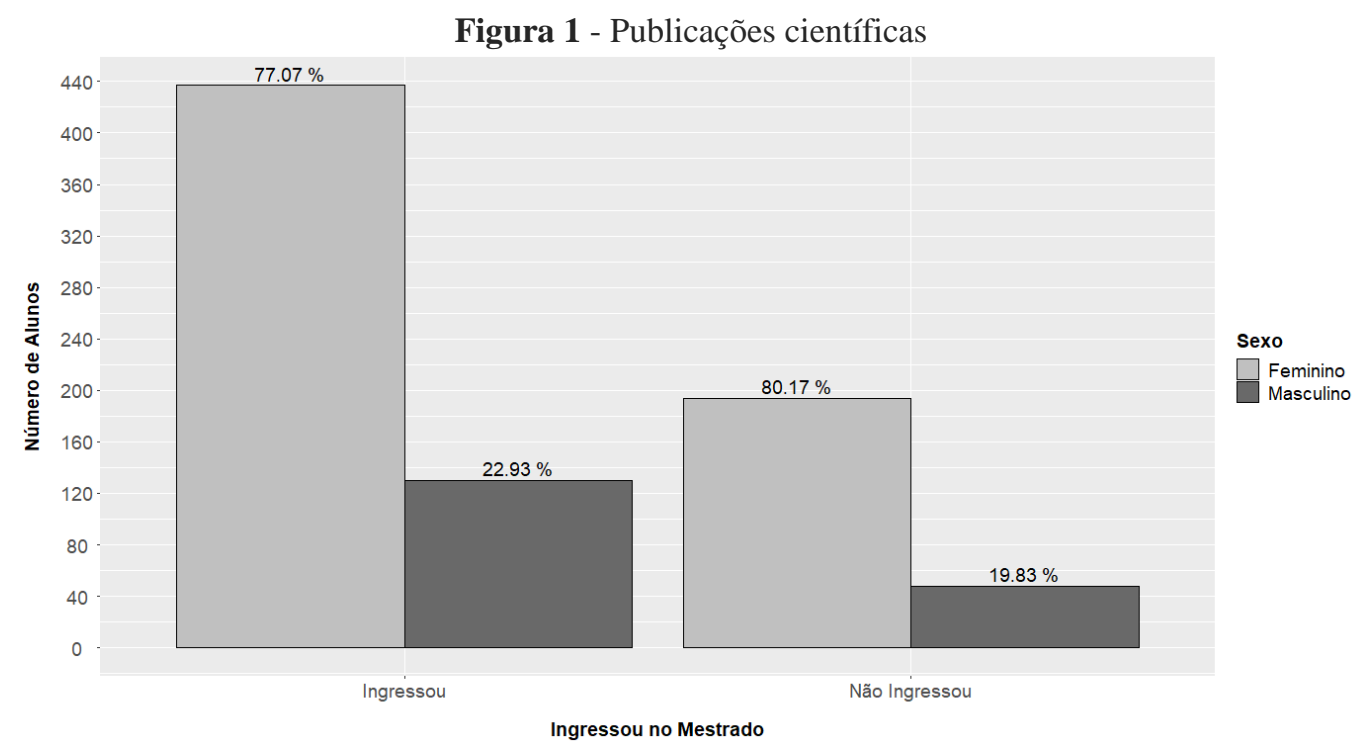

Fonte: dados da pesquisa (2020).

Nota-se que as proporções se mantiveram equiparadas para as categorias de sexo relativas ao encaminhamento para o mestrado - assumindo, portanto, que, entre os participantes de IC nos cursos da área de Saúde da UFPR, o sexo não encontra associação como ingresso no mestrado. 
Por outro lado, ainda quanto ao ingresso em mestrados, registraram-se proporções estatisticamente distintas em relação aos cursos $\left(X^{2}(5)=122,02, p\right.$ valor $<0,000)$, conforme mostrado na Figura 2:

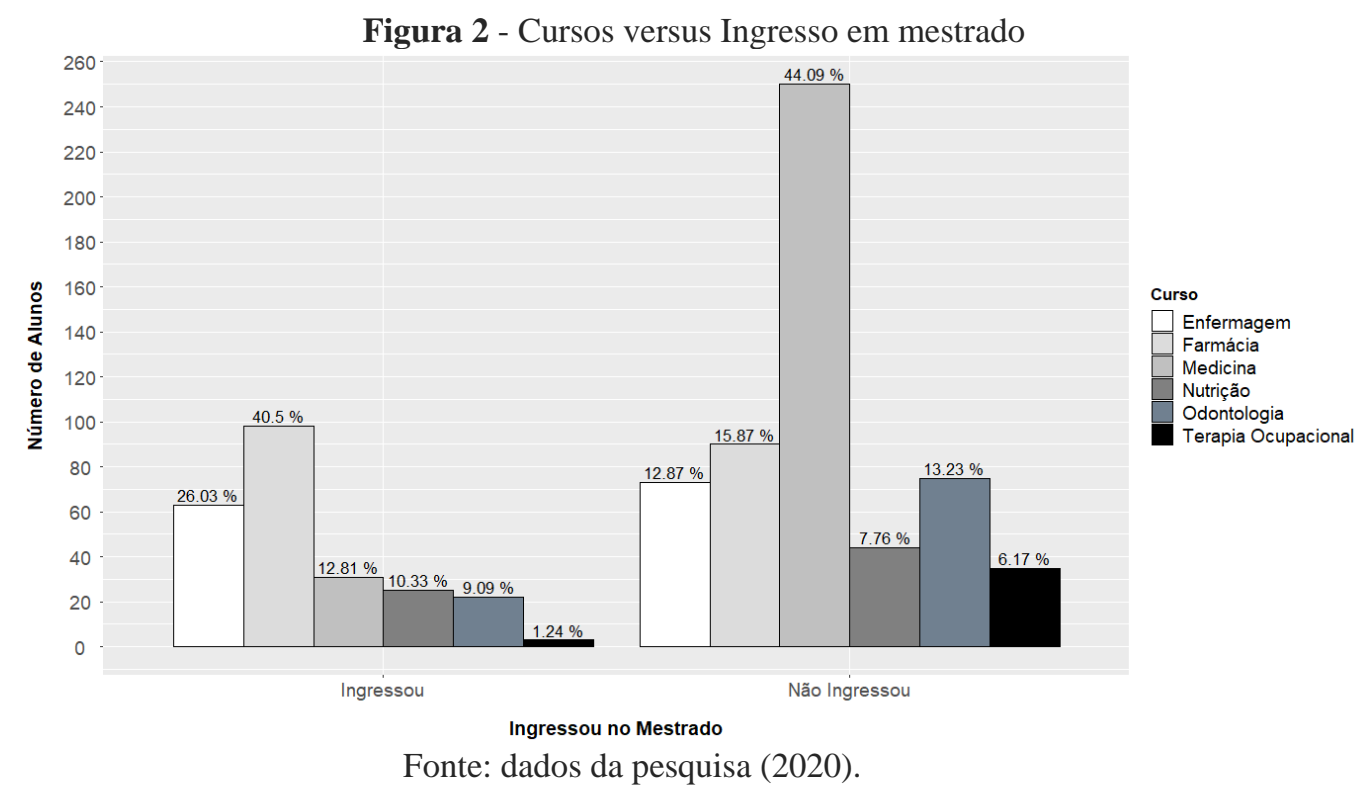

As diferenças apontadas pelo teste se fazem notáveis, e o curso de Farmácia mostrou ser aquele que mais conduz os estudantes para mestrados, totalizando praticamente metade dos estudantes encaminhados para esse tipo de curso. De maneira contrária, Medicina mostrou-se o curso com menor número alunos que avançam os estudos, chegando perto da metade do grupo de alunos não ingressantes.

Para os demais testes descritos no protocolo de análise, há que se verificar o pressuposto da normalidade das variáveis numéricas nas categorias das variáveis nominais, pelo teste $\mathrm{W}$ de Shapiro-Francia. O resultado do teste mostrou que apenas uma das variáveis exibiu distribuição que não a afasta drasticamente de uma distribuição normal, a saber: o tempo para o ingresso em mestrado, no grupo de egressos de Medicina ( $\mathrm{W}=0,939$, $\mathrm{p}$-valor $<0,077$ ) e no grupo de bolsas Fundação Araucária ( $\mathrm{W}=0,837$, p-valor < 0,102). Assim sendo, deste ponto em diante optou-se pelo uso de testes não-paramétricos.

Inicialmente, cruzaram-se os dados contidos nas variáveis "Idade", "Tempo permanecido", "Carga horária total", "Total recebido", "Número participações" e "Numero orientadores", com os dados de "Ingresso em 
mestrado". De maneira geral, essas variáveis se distribuíram nos grupos, conforme mostrado na Tabela 3:

Tabela 3 - Ingressantes e não ingressantes em mestrados

\begin{tabular}{l} 
Variável \\
\hline Idade \\
Tempo permanecido \\
Carga horária total \\
Total recebido \\
Número participações \\
Número orientadores
\end{tabular}

Não ingressantes em mestrado

\begin{tabular}{|c|c|c|c|}
\hline média & desvio & Min & máximo \\
\hline 23,95 & 2,96 & 19 & 50 \\
\hline 13,90 & 8,71 & 1 & 60 \\
\hline 895,85 & 609,21 & 48 & 4352 \\
\hline $3.416,53$ & $6.152,68$ & 0,00 & $20.520,00$ \\
\hline 1,50 & 0,80 & 1 & 6 \\
\hline 1,11 & 0,35 & 1 & 3 \\
\hline
\end{tabular}

Fonte: dados da pesquisa (2020).
Ingressantes em mestrado

\begin{tabular}{rrrr}
\hline média & desvio & min & máximo \\
\hline 23,40 & 2,76 & 19 & 46 \\
16,03 & 8,71 & 1 & 56 \\
$1.076,42$ & 677,78 & 48 & 4224 \\
$4.105,72$ & $3.326,09$ & 0,00 & $15.920,00$ \\
1,85 & 1,01 & 1 & 6 \\
1,11 & 0,33 & 1 & 3 \\
\hline
\end{tabular}

Algumas diferenças médias se destacam: os ingressantes em programas de mestrado permaneceram 15,32\% em número médio de meses a mais do que os alunos que não ingressaram, configurando uma diferença estatisticamente significativa ( $\mathrm{W}=58160$, p-valor $<0,000$ ); por esta razão, a carga horária total em programas de IC foi 20,16\% maior para estes ( $\mathrm{W}=56996$, $\mathrm{p}$-valor $<0,000$ ); e, consequentemente, também o valor total recebido em bolsas, cuja diferença média ( $\mathrm{W}=59448$, p-valor < 0,002) apresentou quase a mesma variação, de 20,17\%. Além disso, também o número médio de participações em programas de IC foi 23,33\% maior para os alunos que, após terem concluído a graduação, optaram pela carreira acadêmica. Por outro lado, não se registraram diferenças estatisticamente significativas quanto ao número de orientadores $(\mathrm{W}=67608$, pvalor < 0,536). É importante relatar que o teste $\mathrm{W}$ de Wilcoxon-Mann-Whitney apontou a existência de diferenças estatisticamente significativas para a idade ( $\mathrm{W}=78636$, p-valor < 0,000), porém, mesmo que se registre uma diferença de $2,35 \%$ entre as duas categorias, não se pode descartar a ocorrência de erro do tipo I.

O próximo conjunto de análises tem como resposta as quantidades de publicações. A primeira delas trata de buscar associação entre "Sexo" e "Publicações durante a IC", conforme exibido na Tabela 4:

Tabela 4 - Sexo versus Número total de publicações durante IC

\begin{tabular}{lrrrrr} 
Sexo & média & desvio & Mínimo & máximo & total \\
\hline Masculino & 4,33 & 5.37 & 0 & 31 & 771 \\
Feminino & 5,16 & 9,12 & 0 & 110 & 3262 \\
\hline
\end{tabular}

Fonte: dados da pesquisa (2020). 
Para este cruzamento de dados, ainda que a média de publicações de mulheres seja maior que o para o grupo de homens, o teste W de WilcoxonMann-Whitney indica o sexo como fator não determinante para o número de publicações $(\mathrm{W}=55081, \mathrm{p}$-valor < 0,692). Na sequência, tem-se a comparação de publicações registradas após os términos dos vínculos com a IC e o sexo, observada na Tabela 5:

Tabela 5 - Sexo versus Número de publicações após IC

\begin{tabular}{lrrrrr} 
Sexo & média & desvio & Mínimo & máximo & total \\
\hline Masculino & 9,11 & 16,58 & 0 & 129 & 1623 \\
Feminino & 9,82 & 20,03 & 0 & 242 & 1623 \\
\hline \multicolumn{5}{c}{ Fonte: dados da pesquisa (2020). }
\end{tabular}

Da mesma forma como encontrado anteriormente, as pequenas diferenças não foram suficientes para que se caracterizassem como estatisticamente significativas $(\mathrm{W}=56794$, $\mathrm{p}$-valor $<0,813)$.

Quanto ao número total de publicações associadas ao fato de o egresso ter se vinculado a mestrados, a Tabela 6 apresenta um resumo descritivo:

Tabela 6 - Ingresso em mestrados versus Número total de publicações durante IC

\begin{tabular}{lrrrrr} 
Ingresso em PPGs & média & desvio & mínimo & máximo & total \\
\hline Não & 3,78 & 6,32 & 0 & 85 & 2.146 \\
Sim & 7,79 & 11,56 & 0 & 110 & 1.887 \\
\hline
\end{tabular}

Fonte: dados da pesquisa (2020).

Entre os egressos de programas de IC que entraram em mestrados, a média do número de publicações foi 2,06 vezes maior do que aqueles que não deram prosseguimento ao mestrado - trata-se de uma diferença estatisticamente significativa $(\mathrm{W}=48658$, $\mathrm{p}$-valor $<0,000)$.

Diferença maior ainda foi encontrada nas publicações após IC, visualizadas na Tabela 7:

Tabela 7 - Ingresso em mestrados versus Número de publicações após IC

\begin{tabular}{lrrrrr} 
Ingresso em PPGs & média & desvio & mínimo & máximo & total \\
\hline Não & 4,66 & 9,64 & 0 & 73 & 2.646 \\
Sim & 21,40 & 28,90 & 0 & 242 & 5.179 \\
\hline \multicolumn{5}{c}{ Fonte: dados da pesquisa (2020). }
\end{tabular}


Nesse caso, a diferença estatisticamente significativa $(\mathrm{W}=31847, \mathrm{p}-$ valor $<0,000$ ) foi de 4,59 vezes maior para a média de publicações dos egressos de IC que entraram em programas de mestrado.

Em relação aos cursos, os números para as publicações totais durante as atividades de IC são mostrados na Tabela 8:

Tabela 8 - Cursos versus Número total de publicações durante IC

\begin{tabular}{lrrrrr}
\hline Cursos & Média & desvio & mínimo & máximo & total \\
\hline Enfermagem & 8,66 & 10,18 & 0 & 70 & 1178 \\
Farmácia & 3,34 & 4,37 & 0 & 19 & 628 \\
Medicina & 3,72 & 4,87 & 0 & 36 & 1048 \\
Nutrição & 2,89 & 3,77 & 0 & 18 & 200 \\
Odontologia & 9,01 & 16,82 & 0 & 110 & 874 \\
Terapia Ocupacional & 2,76 & 3,46 & 0 & 16 & 105 \\
\hline
\end{tabular}

Fonte: dados da pesquisa (2020).

As diferenças estatisticamente significativas encontradas pelo teste $\mathrm{X}^{2} \mathrm{de}$ Kruskal-Wallis $\left(\mathrm{X}^{2}(5)=51,106\right.$, $\mathrm{p}$-valor $\left.<0,000\right)$ dão conta de que egressos do curso de Enfermagem publicaram em média, no mínimo, 2,32 vezes mais que o curso que registrou média de publicações imediatamente inferior (Medicina). Semelhante situação ocorre com Odontologia, que publicou, em média, 2,42 vezes mais do que os egressos de IC do curso de Medicina.

Mesmo cenário é encontrado nas publicações após as atividades de IC, visualizado na Tabela 9:

Tabela 9 - Cursos versus Número de publicações após IC

\begin{tabular}{lrrrrr} 
Cursos & média & desvio & mínimo & máximo & total \\
\hline Enfermagem & 15,25 & 25,45 & 0 & 146 & 2.074 \\
Farmácia & 10,31 & 22,77 & 0 & 242 & 1.940 \\
Medicina & 9,38 & 17,24 & 0 & 129 & 2.637 \\
Nutrição & 5,82 & 8,95 & 0 & 44 & 402 \\
Odontologia & 6,86 & 14,04 & 0 & 73 & 666 \\
Terapia Ocupacional & 2,78 & 6,45 & 0 & 36 & 106 \\
\hline
\end{tabular}

Fonte: dados da pesquisa (2020).

Nesse caso, os destaques também são os egressos de IC do curso de Enfermagem, que publicaram, em média, 47,91\% mais do que o segundo curso que mais publicou (Farmácia) - tais diferenças se configuram como estatisticamente significativas $\left(\mathrm{X}^{2}(5)=25,536\right.$, $\mathrm{p}$-valor $\left.<0,000\right)$.

A última etapa do protocolo de análise proposto trata de buscar relações entre variáveis numéricas explicativas (Idade, Tempo permanecido, Carga 
horária total, Total recebido, Número participações e, Número de orientadores) e respostas (Tempo para ingresso, Publicações durante IC, Publicações durante IC em parceria e, Publicações pós IC). Em relação ao tempo para ingresso em mestrados, a Tabela 10 mostra os resultados para o teste de correlação de Spearman (rho):

Tabela 10 - Variáveis numéricas versus Tempo para ingresso em mestrado

\begin{tabular}{lrr} 
Variável & rho & p-valor \\
\hline Idade & 0,001 & 0,987 \\
Tempo permanecido & $-0,131$ & 0,041 \\
Carga horária total & $-0,094$ & 0,141 \\
Valor total recebido & $-0,116$ & 0,069 \\
Número de participações & $-0,219$ & 0,000 \\
Número de orientadores & $-0,159$ & 0,012 \\
\hline \multicolumn{2}{c}{ Fonte: dados da pesquisa $(2020)}$.
\end{tabular}

Fonte: dados da pesquisa (2020).

Nem todos os resultados mostraram correlações estatisticamente significativas, porém se destaca o fato de o número de participações em atividades de IC apresentar uma relação significativa e inversamente proporcional, indicando que, quanto maior o número de participações em IC, menor é o tempo transcorrido, após a graduação, para o ingresso em mestrados.

Por fim, variáveis explicativas foram associadas aos números de publicações. A Tabela 11 mostra os resultados dessas operações:

Tabela 11 - Variáveis numéricas versus Números de publicações

\begin{tabular}{llrr} 
Variável & Estatísticas & Durante IC & Após IC \\
\hline Idade & rho & 0,007 & $-0,155$ \\
& p-valor & 0,837 & 0,000 \\
\hline \multirow{2}{*}{ Tempo permanecido } & rho & 0,388 & $-0,015$ \\
& p-valor & 0,000 & 0,654 \\
\hline Carga horária total & rho & 0,343 & 0,000 \\
& p-valor & 0,000 & 0,998 \\
\hline Valor total recebido & rho & 0,247 & $-0,080$ \\
& p-valor & 0,000 & 0,021 \\
\hline Número de participações & rho & 0,360 & 0,023 \\
& p-valor & 0,000 & 0,513 \\
\hline Número de orientadores & rho & 0,076 & $-0,131$ \\
& p-valor & 0,030 & 0,000 \\
\hline
\end{tabular}

Fonte: dados da pesquisa (2020).

Com exceção das variáveis "Idade" em relação a "Durante IC" e "Após IC" em relação a "Tempo permanecido", "Carga horária total" e "Número de participações", todos os demais cruzamentos mostraram relações estatisticamente significativas, com destaque para o tempo permanecido em IC 
quando associado com o número total de publicações, cujos rhos obtidos indicam que quanto maior a quantidade de tempo em IC, maior será a quantidade de publicações. É importante destacar que os resultados apontam para o fato de que as variáveis "Tempo permanecido", "Carga horária total", "Número de participações" e "Número de orientadores" possam estar representando o mesmo fenômeno.

Os resultados encontrados merecem ser confrontados com pesquisas anteriores. Quanto ao fato de os egressos que participaram de atividades de IC, ao buscarem mestrados, os terem procurado na própria instituição, tal situação é compreensível, visto que estudantes que atuam junto à IC têm proximidade muito maior com os programas de mestrado das instituições às quais pertencem, sendo esses alunos considerados como os que ingressam mais rapidamente em cursos de mestrado (PINHO, 2017).

Em relação à quantidade de publicações científicas, os números encontrados na presente pesquisa alinham-se à observação de Lopes e Sousa Junior (2018, p. 144), ao apontarem IC como a "[...] possibilidade de o aluno publicar artigos em periódicos e revistas, apresentar pôsteres e trabalhos em semanas de divulgação científica”.

A baixa procura por mestrados pelos egressos de Medicina que, durante a graduação, participaram de atividades de IC não é um fato isolado encontrado na população analisada: Soares, et al., (2017, p. 2), citando Csillag e Schor (1999) chegam a descrever o pesquisador médico como "[...] uma espécie ameaçada de extinção", uma vez que os estudantes de Medicina se deparam com muitas dificuldades para participarem de pesquisas científicas, haja vista a falta de materiais, de estrutura adequada e de pessoal motivado e qualificado nessa área. Indo além, Pirola, et al. (2020, p. 4) apontam como barreira para o ingresso de graduandos de Medicina no "caminho científico" a falta de informações sobre o processo de pesquisa e análise estatística.

$\mathrm{O}$ fato de ingressantes em programas de mestrado terem participado por mais tempo em programas de IC não surpreende: parece ser um comportamento que caracteriza precocemente a inclinação às atividades de pesquisa, como demonstrado nos estudos de Lordelo e Argôlo (2015) e Santos e Leal (2014). 
Porém, não se trata de consenso: ao analisar as características de ingressantes em todos os Programa de Pós-Graduação (PPG) de Ciências Contábeis, mantidos em instituições públicas brasileiras, Miranda et al. (2014) encontraram que apenas 2,9\% haviam frequentado atividades de IC.

Também a ausência de distinções associadas ao sexo quanto aos números de publicações encontra lastro em estudos que discutem esta questão (gênero versus produtividade acadêmica): Mendes et al., (2017), ao estudarem a produção científica de 39 mestres titulados pelo PPG de Ambiente e Sistemas de Produção Agrícola, da Universidade do Estado de Mato Grosso, não encontraram diferenças estatisticamente significativas entre os gêneros; Souza et al. (2017), em uma pesquisa bibliométrica realizada em 113 artigos de periódicos Q1 e Q2, constaram que as diferenças existentes eram não expressivas.

Para o número de publicações para os egressos de IC que deram continuidade aos estudos em nível de mestrado, em média o dobro em relação aos que não seguiram a carreira acadêmica, espera-se, de fato, que esse egresso se torne mestrando com maior veiculação de trabalhos em periódicos, livros, comunicações orais e outros tipos de produção científica (LORDELO; ARGÔLO, 2015).

Em relação aos cursos, em algum momento os resultados se alinham com pesquisas anteriores e em outros, são registradas divergências. Por exemplo, ao se empregar a métrica de número de publicações, o fato de existirem cursos que parecem se beneficiar das atividades de IC, comparados aos demais, já foi constatado por Lordelo e Argôlo (2015). Por outro lado, o destaque do curso de Enfermagem, com números médios de publicações bem acima dos demais cursos do SCS da UFPR, contrasta com a pesquisa conduzida por Santos, Anjos e Almeida (2015), na qual verificaram que a atividade de pesquisa na graduação de 81 estudantes de Enfermagem não mostrou impacto nas publicações daqueles alunos, visto que $96 \%$ dos entrevistados não possuíam nenhum trabalho científico submetido e $99 \%$ dos alunos não tinham qualquer produção publicada.

A relação entre o tempo de ingresso no mestrado e, principalmente, o número de participações em atividades de IC (e o consequente número de 
orientadores, bem como o tempo permanecido) não é um achado inédito: na pesquisa supervisionada por Galvão (2016, p. 156), com 4.634 bolsistas PIBIC que se graduaram na Universidade Estadual Paulista entre 2001 e 2014, verificou-se que a probabilidade de esse bolsista " [...] ingressar no mestrado em até 1 ano é de $72 \%$. No grupo controle, essa probabilidade é menor (63\%), com uma diferença de 9,4 pontos percentuais em relação à primeira"; já o estudo de Ferreira et al. (2015, p. 183), em uma revisão de 1.970 artigos encontrados nas bases Medline, Lilacs e BDenf, constatou que "[...] a motivação para cursar a pós-graduação stricto sensu pode começar ainda no curso de graduação, por meio da inserção, o mais precocemente possível, em programas de iniciação científica".

Por sua vez, o número de publicações desenvolvidas pelos egressos da área da Saúde da UFPR associados ao tempo dedicado para a atividade de IC, bem como o número de participações, encontra ecos no trabalho de pesquisa de Morales, Grineski e Collins (2017), no qual os autores notaram que o aumento no número de publicações em coautoria entre professores e seus estudantes é consequência do processo natural e contínuo da orientação em atividades de pesquisa.

\section{Considerações finais}

Ao tecer as considerações finais sobre a presente investigação, faz-se necessário resgatar o problema norteador ("existem relações entre as características de egressos de IC de cursos da área da Saúde da UFPR quanto à produção científica e à inserção em mestrados?") e o contrapor face aos resultados obtidos. Assim sendo, evidenciaram-se os seguintes resultados:

- Participantes voluntários apresentaram proporção maior dentro do grupo de alunos que não ingressaram no mestrado;

- O curso de Medicina possuiu representatividade consideravelmente maior para o grupo de egressos de IC que não foram encaminhados ao mestrado do que aqueles que deram sequência à carreira acadêmica;

- Egressos que se tornaram mestrandos permaneceram por mais tempo em atividades IC, consequentemente, tendo registrado maior tempo de 
permanência na atividade, maior carga horária calculada e maior montante financeiro acumulado, além de desempenho superior para os três cenários de publicações abordados - durante IC, em parceria e após $\mathrm{IC}$;

- Quanto aos cursos, para o total de publicações desenvolvidas durante a IC, o destaque ficou no curso de Odontologia. Para os trabalhos publicados após IC, o curso de Enfermagem figurou como o mais produtivo;

- Terapia Ocupacional postulou-se como curso de menor produtividade nos dois cenários analisados;

- Para o tempo de ingresso em mestrado, enquanto Farmácia e Odontologia demonstraram menor tempo médio de ingresso de seus estudantes, o curso de Medicina apresentou o maior tempo médio, superior a quatro anos. Considerando o tipo de participação, os bolsistas da Fundação Araucária registraram menor tempo médio para o ingresso em mestrados, porém, há que se ter cautela com este resultado, diante do número baixo de estudantes nessa categoria;

- As publicações realizadas durante IC encontraram relações com o tempo permanecido, carga horária total e quantidade de participações.

Ainda que o objetivo proposto ("identificar padrões entre a atividade de IC associadas às publicações científicas desenvolvidas pelos estudantes e ao ingresso destes em cursos de mestrado") tenha sido atingindo, alguns resultados necessitam que novas abordagens sejam conduzidas para esclarecer algumas situações suscitadas no presente estudo. Por exemplo: (1) por que egressos que atuaram em atividades de IC de determinados cursos são mais propensos a seguirem a carreira acadêmica (especificamente, Farmácia e Enfermagem)? (2) por que os alunos que realizaram atividades de IC do curso de Medicina, quando buscam mestrados, demoram a fazê-lo, em comparação com os egressos dos demais cursos da área de Saúde da UFPR? (3) por que egressos de Odontologia e de Enfermagem chegam a publicar, em média, acima do dobro dos demais cursos? Nesse sentido, sugerem-se pesquisas de cunho qualitativo que permitam identificar a motivação de discentes, docentes e outros atores envolvidos com a 
atividade de IC. As técnicas e os procedimentos presentes nesse tipo de abordagem irão permitir a compreensão de fatores e relações não atingidas pela pesquisa quantitativa.

Independentemente dos resultados encontrados, estes destacam a importância de instrumentos eficazes que possam exprimir o desempenho dos discentes durante a IC. Tais mecanismos possibilitam repasse de recursos financeiros de modo otimizado, cada vez mais necessário, vide o desestimulo constante no incentivo para recursos voltados à pesquisa em território nacional. Ainda que a baixa progressão para cursos de mestrado tenha sido o resultado mais notável da investigação, não se deve desconsiderar a atratividade que outras modalidades, como cursos de especialização e principalmente as residências médicas e multiprofissionais, possuem para os egressos dessa área. Os resultados encontrados apontam para a necessidade de reflexão sobre as atividades desenvolvidas no âmbito acadêmico, sobretudo a pesquisa científica. Tratando em especial dos cursos da área da Saúde, os currículos devem ser respaldados com maior incentivo, carga horária específica e abordagens de ensino e aprendizado que favoreçam o desenvolvimento de práticas voltadas à pesquisa científica. Executar tais ações favorecerá o surgimento de novos profissionais diferenciados, com capacidade de contribuir e desenvolver a área da Saúde, independentemente do prosseguimento no caminho da pesquisa científica.

\section{Referências}

ANDERSON, David R; SWEENEY, Dennis J; WILLIAMS, Thomas A; CAMM, Jeffrey D; COCHRAN, James J. Statistics for business \& economics. 13. ed. Boston: Cengage Learning, 2017.

BAZIN, Maurice Jacques. O que é iniciação científica? Revista de Ensino de Física, São Paulo, v. 5, n. 1, p. 81-88, jun. 1983.

BRASIL. MINISTÉRIO DA SAÚDE. Agenda nacional de prioridades de pesquisa em Saúde. 2. ed. Brasília: Ministério da Saúde, 2015.

BRAVO, Bianca Camila; CHAUD, Daniela Maria Alves; ABREU, Edeli Simioni. Avaliação da motivação acadêmica de universitários do curso de 
Nutrição de uma universidade privada de São Paulo. Simbio-Logias, Botucatu, v. 6, n. 9, p. 57-72, dez. 2013.

CABRERO, Rodrigo Castro; COSTA, Maria Piedade Resende. Iniciação científica, bolsa de iniciação científica e grupos de pesquisa. In: MASSI, Luciana; QUEIROZ, Salete Linhares. Iniciação científica: aspectos históricos, organizacionais e formativos da atividade no ensino superior brasileiro. São Paulo: Editora UNESP, p. 109-130, 2015.

CONSELHO NACIONAL DE DESENVOLVIMENTO CIENTÍFICO E TECNOLÓGICO. Resolução normativa 017/2006. Ministério da Ciência, Tecnologia, Inovações e Comunicações. Brasília: CNPQ. 2006.

CSILLAG, Cláudio; SCHOR, Nestor. O pesquisador médico: da academia as parceiras. Revista da Associação Médica Brasileira, São Paulo, v. 45, n. 2, p. 152-156, 1999.

ERDMANN, Alacoque Lorenzini; LEITE, Joséte Luzia; NASCIMENTO, Keyla Cristiane; LANZONI, Gabriela Marcellino Melo. Vislumbrando a iniciação científica a partir das orientadoras de bolsistas da Enfermagem. Revista Brasileira de Enfermagem, Brasília, v. 64, n. 2, p. 261-267, mar./abr. 2011.

FERREIRA, Rejane Eleuterio; TAVARES, Cláudia Mara Melo; SANTOS, Gabriela Silva; FONSECA, Paula Isabella Marujo Nunes. Motivação de enfermeiro para ingressar em uma pós-graduação stricto sensu. Revista Baiana de Enfermagem, Salvador, v. 29, n. 2, p. 180-185, abr./jul. 2015.

FIELD, Andy; MILES, Jeremy; FIELD, Zoe. Discovering statistics using R. London: Sage, 2012.

FIGUEIREDO, Wesley Pereira Santos; NUNES, Tiago Silva; MOURA, Tauanny Aragão; LIMA, Rosiane Santana Andrade; TANAJURA, Diego Moura. Pesquisa científica: conhecimento, atitudes e barreiras entre estudantes de medicina brasileiros. Archives of Health Sciences, São José do Rio Preto, v. 25, n. 3, p. 56-59, dez. 2018.

GALVÃO, Antonio Carlos Figueira. A formação de novos quadros para CT\&I: avaliação do Programa Institucional de Bolsas de Iniciação Científica - PIBIC. Centro de Gestão e Estudos Estratégicos. Brasília: CGEE, p. 1-175. 2016.

GROSS, J.; LIGGES, U. Package 'nortest', 2019. Disponível em: <https://cran.r-project.org/web/packages/nortest/nortest.pdf>. Acesso em: 6 dezembro 2020.

KING, Andrew; ECKERSLEY, Robert. Statistics for biomedical engineers and scientists. London: Academic Press, 2019. 
KLOKE, John; MCKEAN, Joseph W. Nonparametric statistical methods using R. Boca Raton: CRC Press, 2014.

KRASKA-MULLER, Marie. Nonparametric statistics for social and behavioral sciences. Boca Raton: CRC Press, 2019.

KRUSKAL, William Henry; WALLIS, Wilson Allen. Use of ranks in onecriterion variance analysis. Journal of the American Statistical Association, Washington, v. 47, n. 260, p. 583-621, december 1952.

LOPES, Maria Janice Pereira; SOUSA JÚNIOR, Dárcio Luiz. Iniciação científica: uma análise de sua contribuição na formação acadêmica. Revista Cesumar, Maringá, v. 23, n. 1, p. 133-148, 2018.

LORDELO, José Albertino Carvalho; ARGÔLO, Rodrigo Ferrer. Influências da iniciação científica na pós-graduação. Estudos em Avaliação Educacional, São Paulo, v. 26, n. 61, p. 168-191, jan./abr. 2015.

MANN, Henry Bertold; WHITNEY, Donald Ransom. On a test of whether one of two random variables is stochastically larger than the other. The Annals of Mathematical Statistics, Detroit, v. 18, n. 1, p. 50-60, 1947.

MASSI, Luciana; QUEIROZ, Salete Linhares. Iniciação Científica: aspectos históricos, organizacionais e formativos da atividade no ensino superior brasileiro. São Paulo: Editora UNESP, 2015.

MATLOFF, Norman. Probability and statistics for data science. Boca Raton: CRC Press, 2019.

MENDES, Inês Roeder Nogueira; ASCARI, João Paulo; BORGES, Janaína Domingos; TOLEDO, Martins; GALVANIN, Edinéia Aparecida Santos. Análise do perfil dos mestrandos do Programa de Pós-Graduação Ambiente e Sistemas de Produção Agrícola. Nucleus, Ituverava, v. 14, n. 1, p. 267-278, abr. 2017.

MIRANDA, Gilberto José; LEMES, Sirlei; LIMA, Francielly Dornelas Correia; BRUNO JÚNIOR, Vicente. Relações entre desempenho acadêmico e acesso aos programas de mestrado em Ciências Contábeis. Revista Ambiente Contábil, Natal, v. 6, n. 1, p. 141-162, jan./jun. 2014.

MORALES, Danielle X; GRINESKI, Sara E; COLLINS, Timothy W. Increasing research productivity in undergraduate research experiences: exploring predictors of collaborative faculty-student publications. CBE - Life Sciences Education, Bethesda, v. 16, n. 3, p. 1-9, fall 2017.

NARDINI, Elisa Fonseca; TURSSI, Cecilia Pedroso; SILVA, Almenara Souza Fonseca; FLÓRIO, Flávia Martão. Política de estímulo à iniciação científica: 
impacto no coeficiente de rendimento de graduandos em Odontologia. Revista da ABENO, Porto Alegre, v. 19, n. 1, p. 33-39, jan./mar. 2019.

OGLE, Derek; WHEELER, Powell; DINNO, Alexis. Simple Fisheries Stock Assessment Methods. [S.1.]: The Comprehensive R Archive Network, 2019. PEARSON, Karl. On the criterion that a given system of deviations from the probable in the case of a correlated system of variables is such that it can be reasonably supposed to have arisen from random sampling. Philosophical Magazine Series 5, Oxfordshire, v. 50, n. 302, p. 157-175, 1900.

PINHO, Maria. José. Ciência e ensino: contribuições da iniciação científica na educação superior. Avaliação, Sorocaba, v. 22, n. 3, p. 658-675, nov. 2017.

PINTO, Natália Lúcia Silva; FERNANDES, Laura Maria Abdon; SILVA, Fabiana Ferreira. Para além da formação acadêmica: as contribuições da iniciação científica para o desenvolvimento pessoal e profissional de estudantes da área de Administração. Administração: Ensino e Pesquisa, São Paulo, v. 17, n. 2, p. 301-325, mai./ago. 2016.

PIROLA, Sarah Beatriz França Bortolato; PADILHA, Francieli Dias; DI MAURO, Jadna Madureira Bitencourt; PIROLA, Lucas Heitor França Bortolato; GABRIEL, Sthefano Atique. A importância da Iniciação Científica na graduação de Medicina. Revista Corpus Hippocraticum, São José do Rio Preto, v. 1, n. 1, p. 1-7, 2020.

ROCHA, José Gladistone; RODRIGUES, Carlo Kleber Silva. Projeto de Iniciação Científica: uma proposta de processo. Universitas Gestão e TI, Brasília, v. 7, n. 1-2, p. 93-104, jan./dez. 2017.

ROCHA, Lucas Barone; OLIVEIRA, Iuri Pimenta; SILVA, Mayra Oliveira Maciel; REZENDE, Marianna Camilo; SANTOS, Raíssa Aparecida Silva; COELHO, Camila Magalhães; AMÂNCIO, Natália Fátima Gonçalves. Iniciação científica no curso de Medicina: contribuições e desafios. Brazilian Journal of Health Review, Curitiba, v. 2, n. 6, p. 5439-5444, nov./dez. 2019.

SANTOS, Cassius Klay Silva; LEAL, Edvalda Araújo. A iniciação científica na formação dos graduandos em Ciências Contábeis: um estudo em uma instituição pública do triângulo mineiro. Revista Contemporânea de Contabilidade, Florianópolis, v. 11, n. 22, p. 25-48, jan./abr. 2014.

SANTOS, Nicole Cristiane Gica; MELLO, Adilson Silva; CHIMENDES, Vanessa Cristhina Gatto; PAIVA, Cássia Mara Ribeiro. Ciência, tecnologia e sociedade na iniciação científica: o caso de uma universidade do sul de Minas. Perspectivas Contemporâneas, Campo Mourão, v. 12, n. 3, p. 17-32, set./dez. 2017. 
SANTOS, Vanessa Cruz; ANJOS, Karla Ferraz; ALMEIDA, Obertal Silva. Iniciação científica a partir de estudantes de Enfermagem. Revista Brasileira de Ciências da Saúde, João Pessoa, v. 19, n. 4, p. 255-260, 2015.

SHAPIRO, Samuel Sanford; FRANCIA, R. S. An approximate analysis of variance test for normality. Journal of the American Statistical Association, Washington, v. 67, p. 215-216, 1972.

SILVA JUNIOR, Manoelito Ferreira; ASSIS, Rahyza Inácio Freire; SOUSA, Hedilberto Araújo; MICLOS, Paula Vitali; GOMES, Maria José. Iniciação científica: percepção do interesse de acadêmicos de Odontologia de uma universidade brasileira. Saúde e Sociedade, São Paulo, v. 23, n. 1, p. 325-335, 2014.

SILVA, Ítalo Rodolfo; LEITE, Joséte Luzia; TREVIZAN, Maria Auxiliadora; MENDES, Isabel Amélia Costa; SILVA, Thiago Privado; LINS, Silvia Maria Sá Basílio. Aprender pela pesquisa: do ensino da ciência ao campo assistencial da Enfermagem. Escola Anna Nery, Rio de Janeiro, v. 21, n. 4, p. 1-8, 2017.

SOARES, Audrey Cabral Branches; FERREIRA, Iago Gonçalves; CARREIRA, Luciana Brandão; RIBEIRO, Thaís Cortez Silva. Perfil científico de estudantes de Medicina em uma universidade pública. Scientia Medica, Porto Alegre, v. 27, n. 2, p. 1-6, 2017.

SOUZA, Daniele Cristina Ferreira; GALDIN, Josiane; ENDO, Sabrina Arissa Nakano; CRUZ, Cássia Vanessa Olak Alves. Pesquisa contábil no contexto do terceiro setor: análise do perfil das publicações acadêmicas em periódicos internacionais. $2^{\circ}$ CONGRESSO UFU DE CONTABILIDADE, 2, p. 1-17, 2017, Uberlândia. Anais... Uberlândia - UFU, 2017.

SPEARMAN, Charles. The proof and measurement of association between two things. The American Journal of Psychology, Chicago, v. 15, n. 1, p. 72-101, january 1904.

UNIVERSIDADE FEDERAL DO PARANÁ. Iniciação Científica: o processo de institucionalização na UFPR. Curitiba: Pró-Reitoria de Pós-Graduação, 1998.

WILCOXON, Frank. Individual comparisons by ranking methods. Biometrics Bulletin, Washington, v. 1, n. 6, p. 80-83, december 1945.

ZAMPIERI, Viviane Helena; SANTIN, Julio Cesar; SOUSA, Keylla Mara Cardoso; LOPES, Humbelina Silva Siqueira; SANCHES, Sueli; SILVA, Hidelvani Nunes; ISHIKAWA, Aline Akemi; ALMEIDA, Ana Paula Ferreira; PONTES, Felipe Moura. Contribuições da iniciação científica no aprendizado e desenvolvimento dos estudantes no ensino superior. Nativa, Sinop, v. 7, n. 1, p. 74-81, 2018. 


\title{
Scientific publications and access to master's degrees for Undergraduate Research graduates: a study in the courses in the Health Sector at the Federal University of Paraná
}

\begin{abstract}
The study seeks relationships between scientific publications and entry into master's programs of 809 undergraduate research graduates from the Health Sector courses at the Federal University of Paraná (Nursing, Pharmacy, Medicine, Nutrition, Dentistry and Occupational Therapy), between 2002 and 2017. This is a study that employs non-parametric bivariate statistical analysis on data collected from the undergraduate research system of Federal University of Paraná and Lattes platform. The results show that graduates who stayed longer in undergraduate research programs became masters students more quickly, at the same time that they presented a greater number of publications; in addition, participants in the Dentistry and Nursing courses obtained an average number of publications more than double that of the other courses, as well as the graduates of Pharmacy and Nursing followed the academic career more quickly.
\end{abstract}

Keywords: Undergraduate research. Health Sciences. Scientific publications. Masters studies.

Recebido: 10/09/2020

Aceito: 19/01/2021

\section{Declaração de autoria}

Concepção e elaboração do estudo: Felipe Correa Fiori

Coleta de dados: Felipe Correa Fiori

Análise e discussão de dados: Felipe Correa Fiori, Cicero Aparecido Bezerra

Redação e revisão do manuscrito: Cicero Aparecido Bezerra, Márcio Rogério de Souza

\section{Como citar}

FIORI, Felipe Correa; BEZERRA, Cicero Aparecido; SOUZA, Márcio Rogério de. Publicações científicas e acesso a mestrados de alunos de Iniciação Científica: um estudo nos cursos do Setor de Saúde da Universidade Federal do Paraná. Em Questão, Porto Alegre, v. 27, n. 3, p. 204-227, 2021. Doi: http://dx.doi.org/10.19132/1808-5245273.204-227 